Corresponding author: sjones@bcgsc.ca

(c) 2019 Williamson et al. This article is distributed under the terms of the Creative Commons Attribution-NonCommercial License, which permits reuse and redistribution, except for commercial purposes, provided that the original author and source are credited.

Ontology terms: neoplasm of the pancreas; neuroendocrine neoplasm

Published by Cold Spring Harbor Laboratory Press

doi:10.1101/mcs.a003814

\section{Genomic characterization of a well- differentiated grade 3 pancreatic neuroendocrine tumor}

\author{
Laura M. Williamson, ${ }^{1}$ Michael Steel ${ }^{2}$ Jasleen K. Grewal, ${ }^{1}$ My Lihn Thibodeau, ${ }^{1,3}$ \\ Eric Y. Zhao, ${ }^{1}$ Jonathan M. Loree, ${ }^{4}$ Kevin C. Yang, ${ }^{1,5}$ Sharon M. Gorski, ${ }^{1,5}$ \\ Andrew J. Mungall, ${ }^{1}$ Karen L. Mungall, ${ }^{1}$ Richard A. Moore, ${ }^{1}$ Marco A. Marra, ${ }^{1,3}$ \\ Janessa Laskin, ${ }^{4}$ Daniel J. Renouf, ${ }^{4,6,7}$ David F. Schaeffer, ${ }^{2,6,7}$ \\ and Steven J.M. Jones ${ }^{1,3,5,7}$

\begin{abstract}
${ }^{1}$ Canada's Michael Smith Genome Science Centre, BC Cancer, Vancouver, British Columbia V5Z 4S6, Canada; ${ }^{2}$ Department of Pathology \& Laboratory Medicine, Vancouver General Hospital, Vancouver, British Columbia V6T 2B5, Canada; ${ }^{3}$ Department of Medical Genetics, University of British Columbia, Vancouver, British Columbia V6H 3N1, Canada; ${ }^{4}$ Division of Medical Oncology, BC Cancer, Vancouver, British Columbia V5Z 4E6, Canada; ${ }^{5}$ Department of Molecular Biology and Biochemistry, Simon Fraser University, Burnaby,

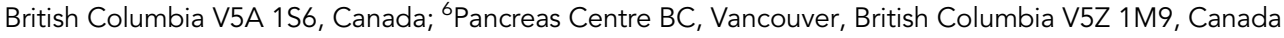

\begin{abstract}
Pancreatic neuroendocrine neoplasms (PanNENs) represent a minority of pancreatic neoplasms that exhibit variability in prognosis. Ongoing mutational analyses of PanNENs have found recurrent abnormalities in chromatin remodeling genes (e.g., DAXX and ATRX), and mTOR pathway genes (e.g., TSC2, PTEN PIK3CA, and MEN1), some of which have relevance to patients with related familial syndromes. Most recently, grade 3 PanNENs have been divided into two groups based on differentiation, creating a new group of well-differentiated grade 3 neuroendocrine tumors (PanNETs) that have had a limited whole-genome level characterization to date. In a patient with a metastatic well-differentiated grade 3 PanNET, our study utilized whole-genome sequencing of liver metastases for the comparative analysis and detection of single-nucleotide variants, insertions and deletions, structural variants, and copy-number variants, with their biologic relevance confirmed by RNA sequencing. We found that this tumor most notably exhibited a TSC1-disrupting fusion, showed a novel CHD7-BEND2 fusion, and lacked any somatic variants in ATRX, DAXX, and MEN1.
\end{abstract}

[Supplemental material is available for this article.]

\section{INTRODUCTION}

Pancreatic neuroendocrine neoplasms (PanNENs) are neoplasms of the endocrine pancreas. These tumors account for $3 \%-5 \%$ of all pancreatic malignancies and in general have a better prognosis than typical pancreatic exocrine malignancies. Positive outcomes are largely dependent on complete surgical excision, as 5-yr survival approaches $55 \%$ when tumors are localized and resected, in contrast to 15\% when they are not resectable (Edge et al. 2010; Dasari et al. 2017). Although PanNENs can closely approximate the morphology of neuroendocrine tumors of the gastrointestinal tract, their biologies differ, and it is, therefore, clinically

\footnotetext{
${ }^{7}$ These authors contributed equally to this work.
} 
advantageous to investigate alternative diagnostic and treatment approaches (Kulke et al. 2011).

PanNEN classification uses tumor morphology and grade to partition well-differentiated from poorly differentiated entities (Klimstra et al. 2010). Poorly differentiated pancreatic neuroendocrine carcinomas (PanNEC) are characterized by both a high mitotic count (>20 per 10 high power field) and a high proliferation index (Ki-67 > 20\%). Well-differentiated pancreatic neuroendocrine tumors (PanNETs) tend to have lower mitotic rates and proliferation indices, classified into low-grade (G1) or intermediate-grade (G2) on the basis of their Ki-67 staining profiles $(<3 \%$ and between $3 \%$ and $20 \%$ of tumor cells, respectively). It is also now recognized that a less-common subset of morphologically well-differentiated PanNETs has an incongruously high proliferation index (Ki-67 > 20\%). Despite their high proliferative rate, these tumors appear to be separate biologic entities from the poorly differentiated NECs, so are now classified as high-grade (G3) PanNETs (Coriat et al. 2016; Tang et al. 2016; Lloyd et al. 2017).

Recurring molecular aberrations in PanNETs have been described in studies using wholeexome and whole-genome sequencing. Namely, mutations in DAXX and ATRX (chromatin remodeling genes) and TSC2, PTEN, PIK3CA, and MEN1 (mTOR pathway genes) have been observed (Jiao et al. 2011; Scarpa et al. 2017; Wong et al. 2018). Moreover, individuals exhibiting germline mutations in TSC1/2 (tuberous sclerosis), MEN1 (multiple endocrine neoplasia 1), VHL (von Hippel-Lindau), and NF1 (neurofibromatosis type 1) are at increased risk of developing PanNETs (Jensen et al. 2008).

Scarpa et al. (2017) provide a large-scale whole-genome landscape of PanNETs, which included five G3 PanNETs in their analysis, but the literature to date has an otherwise limited characterization of molecular aberrancy in these tumors. To this end, this report represents a complete genomic and transcriptomic characterization of a patient with a metastatic welldifferentiated G3 PanNET.

\section{RESULTS}

\section{Clinical Presentation}

A previously healthy 35-yr-old male presented with weight loss and pain. Imaging demonstrated a mass in the pancreatic tail and liver metastasis. The patient was referred to the Personalized OncoGenomics Program (POG) for whole-genome and transcriptome analysis (NCT02155621) (Laskin et al. 2015). A core needle biopsy of one of the liver lesions revealed a G3 well-differentiated neuroendocrine tumor (Ki-67 =30\%; chromogranin- and CK7positive; CK20-negative; octreotide scan-negative) (Fig. 1).

\section{Genomic Analysis}

Whole-genome sequencing of liver metastases and blood was performed for comparative analysis and detection of somatic single-nucleotide variants (SNVs), insertion and deletions (indels), structural variants, and copy-number variants. RNA sequencing was performed to support variants detected by genomic sequencing and identify altered gene expression. Tumor content, as based on the tumor/normal sequencing ratio and loss of heterozygosity, was estimated to be $95 \%$ and a diploid model was used to describe the observed copynumber changes.

\section{Single-Nucleotide Variants}

Comparative analysis of whole-genome sequencing from tumor and normal samples revealed 4232 somatic SNVs. Somatic SNVs were compared to a catalog of previously 

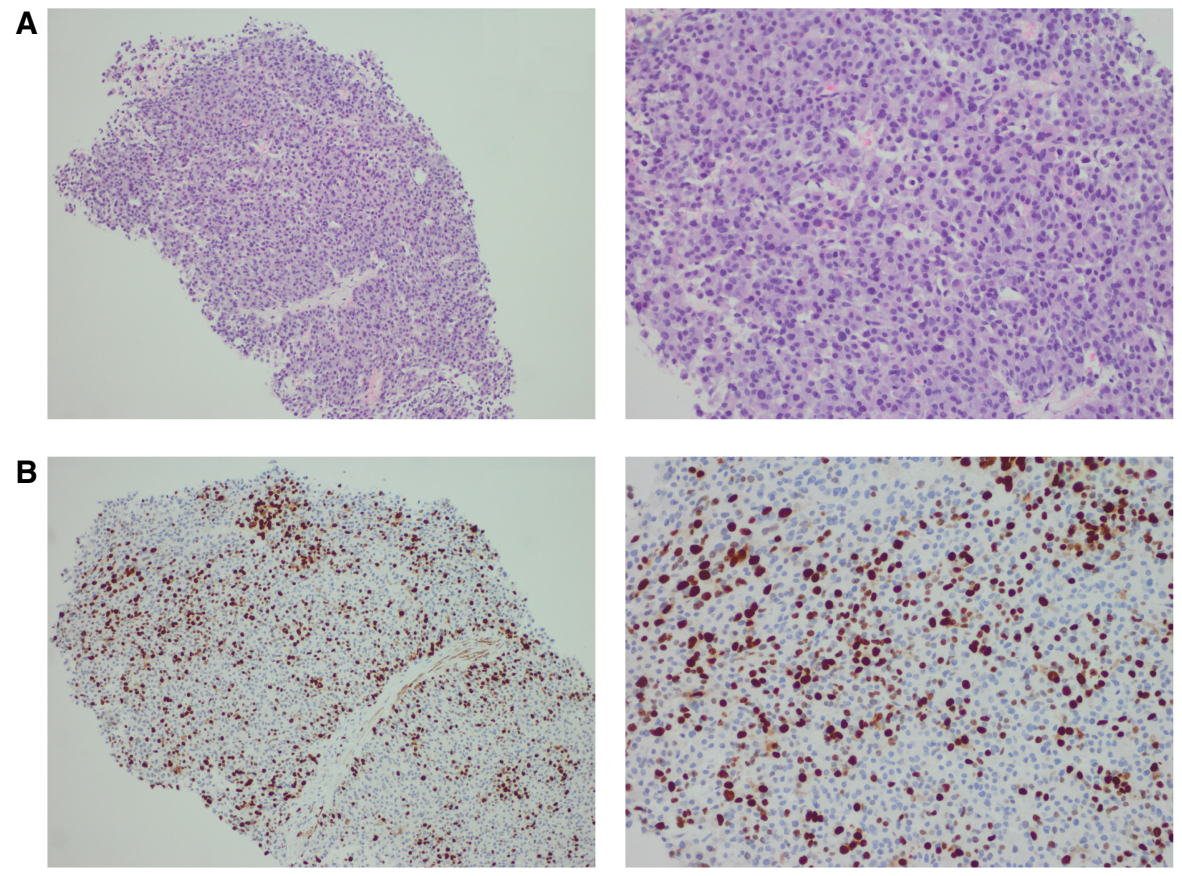

Figure 1. Tumor histopathology and Ki-67 immunohistochemistry. (A) Core biopsy of a liver metastasis stained for hematoxylin and eosin showing a metastatic well-differentiated neuroendocrine tumor clinicoradio-

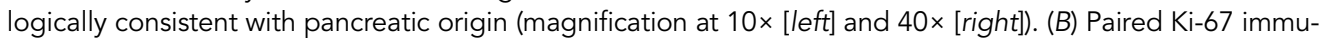
nohistochemistry showing a proliferative index of $30 \%$, classifying the tumor as a well-differentiated grade 3 (G3) neuroendocrine tumor (magnification at $10 \times[l$ eft $]$ and $40 \times[$ right]).

described mutation signatures (http://cancer.sanger.ac.uk/cosmic/signatures) using the signIT package (https://github.com/eyzhao/SignIT/; EY Zhao, E Pleasance, and M Jones, in prep.). COSMIC mutation signature 5 was the most dominant mutation signature (Supplemental Table S1; Supplemental Fig. S1), with 30.8\% (95\% interval 17.7\%-43.4\%) of somatic SNVs attributed to this signature. Signature 5 was also detected as a dominant signature in the majority of ICGC PanNETs indicating a common etiology of mutagenesis across PanNETs (Scarpa et al. 2017). There were 15 somatic nonsynonymous mutations affecting protein-coding genes detected in the tumor biopsy, seven of which were subclonal (Table 1). Despite being recurrently mutated in PanNETs, we did not identify mutations affecting DAXX, ATRX, or MEN1 in this G3 PanNET sample. Similarly, none of the five G3 PanNETs from the ICGC study harbored mutations in DAXX, ATRX, or MEN1.

\section{Copy-Number Alterations}

The genome was subject to loss of one copy each of Chromosome 9 and 13 and broad deletions affecting Chromosomes 1, 2, and 6 (Fig. 2A). Combinatorial analysis of copy-number aberrations (CNAs) and SNVs did not identify loss of heterozygosity of any of the altered protein-coding genes. Homozygous deletion of CDKN2A and CDKN2B on Chromosome 9 and a single-copy loss of RB1 were the main copy alterations of note. Comparison of RB1 expression in this tumor sample with RB1 expression across a compendium of all TCGA data sets as well as the average expression across all tissue types in the lllumina BodyMap reference RNA-seq data set was performed. Expression of RB1 was in the 6th percentile compared to TCGA compendium and down-regulated 3.48-fold compared to the lllumina BodyMap, indicating low expression of RB1 in this sample. 
Table 1. Somatic nonsynonymous single-nucleotide variants (SNVs) and indels

\begin{tabular}{|c|c|c|c|c|c|c|c|c|c|c|}
\hline Gene & $\mathrm{Chr}$ & Position & Ref & Alt & HGVS codons & HGVS protein & Type & Genotype & Predicted Effect & $\mathrm{dbSNP}$ \\
\hline HSPG2 & 1 & 22149892 & G & A & c. $13093 C>T$ & p.Arg 4365* & SNV & Heterozygous & Stop gain & - \\
\hline CNR2 & 1 & 24202047 & G & $\mathrm{T}$ & c. $61 \mathrm{C}>\mathrm{A}$ & p.Pro21Thr & SNV & Heterozygous & Missense & - \\
\hline DNAJC13 & 3 & 132182654 & A & $\mathrm{T}$ & c. $1885 \mathrm{~A}>\mathrm{T}$ & p.Thr629Ser & SNV & Heterozygous & Missense & - \\
\hline MUC7 & 4 & 71347240 & T & C & c. $779 \mathrm{~T}>\mathrm{C}$ & p.Val260Ala & SNV & Subclonal & Missense & rs145745951 \\
\hline RAPGEF2 & 4 & 160279276 & A & C & c. $4485 \mathrm{~A}>C$ & p.Gln1495His & SNV & Heterozygous & Missense & - \\
\hline$F R G 1$ & 4 & 190876269 & $\mathrm{~T}$ & C & c. $395 \mathrm{~T}>\mathrm{C}$ & p.lle132Thr & SNV & Subclonal & Missense & rs149113074 \\
\hline DPCR 1 & 6 & 30918448 & $\mathrm{~T}$ & C & c. $2207 \mathrm{~T}>\mathrm{C}$ & p.Phe736Ser & SNV & Subclonal & Missense & - \\
\hline PTPN6 & 12 & 7069329 & C & $\mathrm{T}$ & c. $1514 C>T$ & p.Ala505Val & SNV & Subclonal & Missense & rs185835888 \\
\hline AHNAK2 & 14 & 105418798 & $\mathrm{~T}$ & C & c. $2990 A>G$ & p.Lys997Arg & SNV & Subclonal & Missense & rs200638785 \\
\hline TXNDC2 & 18 & 9887371 & C & A & c. $895 \mathrm{C}>\mathrm{A}$ & p.Leu299|le & SNV & Subclonal & Missense & rs200059339 \\
\hline ZNF57 & 19 & 2917901 & A & G & c. $1282 A>G$ & p.Thr428Ala & SNV & Subclonal & Missense & - \\
\hline GRIK5 & 19 & 42507823 & C & $\mathrm{T}$ & c. $2276 \mathrm{G}>\mathrm{A}$ & p.Arg759Gln & SNV & Heterozygous & Missense & - \\
\hline SYNJ1 & 21 & 34004062 & A & G & c. 3965 T >C & p.lle1322Thr & SNV & Heterozygous & Missense & - \\
\hline PLCXD1 & $x$ & 215957 & C & CG & c.928dupG & p.Val310fs & Insertion & Heterozygous & Frameshift & - \\
\hline USP7 & 16 & 8989553 & $\begin{array}{l}\text { CTTCT } \\
\text { TGAT }\end{array}$ & CATAA & $\begin{array}{l}\text { c.2858_2865delAT } \\
\text { CAAGAAinsTTAT }\end{array}$ & p.Gln954fs & Indel & Heterozygous & Frameshift & \\
\hline
\end{tabular}

A

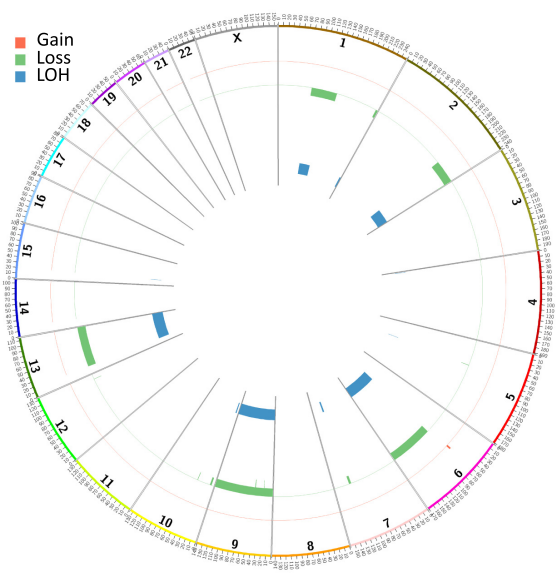

B

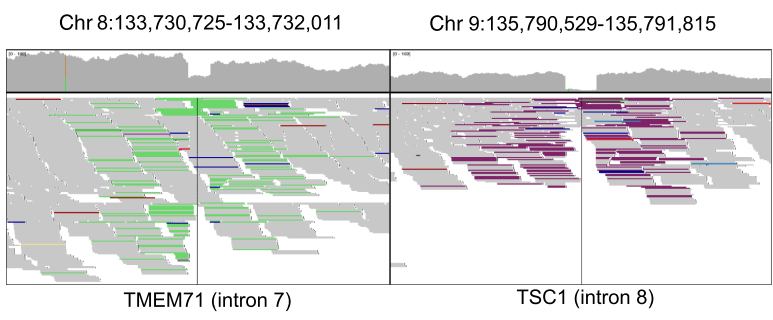

C

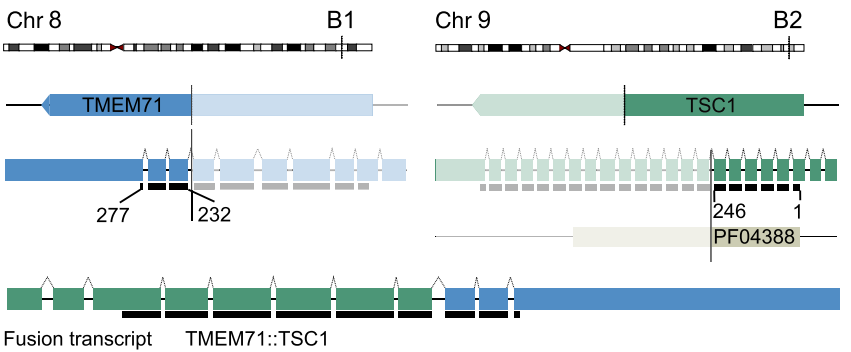

Figure 2. Copy-number aberration and TSC1-TMEM71 structural rearrangement in the G3 PanNET. (A) Circos plot illustrating copy-number alterations and loss of heterozygosity ( $\mathrm{LOH}$ ) observed in the tumor sample. (B) Genomic sequencing read alignment of the genomic regions affected by the rearrangement. Green reads that aligned to TSC1 and purple reads that aligned to TMEM71 indicate split reads that support the rearrangement. (C) Illustration of TSC1-TMEM71 rearrangement generated by MAVIS (Reisle et al. 2018). The TMEM71 and TSC1 breakpoints are indicated at the chromosome, gene, and transcript level (B1 and B2, respectively, upper box). Protein-coding sequence associated with the respective transcripts are indicated by the black line and the amino acids included in the fusion product are indicated. Pfam (http://pfam.xfam.org) protein domains are indicated in the track below the transcript: Hamartin (PF04388). The predicted fusion transcript and protein-coding sequence are shown in the lower box (TSC1 exons are colored green; TMEM71 exons are colored blue). 


\section{TSC1-TMEM71 Rearrangement}

Structural variants were identified using de novo assembly followed by variant detection. A novel somatic translocation between Chromosomes 8 and 9 (t(9;8)(q34.13;q24.22)) was detected in the genomic assembly (Fig. 2B) and supported by the transcriptomic assembly. The rearrangement resulted in an in-frame fusion of TSC1 exons 1-8 (encoding amino acids 1246) with TMEM71 exons 8-10 (encoding amino acids 232-277) (Fig. 2C). TSC1 encodes the tumor suppressor, hamartin. Hamartin heterodimerizes with the GTPase, tuberin, encoded by the TSC2 gene. The TSC1/2 protein complex suppresses cell growth, largely by inhibiting the small G-protein Rheb, a crucial activator of the mTORC1 pathway (Castro et al. 2003; Inoki et al. 2003). The predicted TSC1-TMEM71 fusion protein identified in this tumor lacks protein sequence critical for TSC2 binding and is thus predicted to disrupt TSC1 function (Huang and Manning 2008). Deletion of exon 9 alone has been shown to disrupt TSC1TSC2 dimer formation and downstream inhibition of mTORC1 kinase activity (Santiago Lima et al. 2014), supporting this fusion as indeed a loss of function variant. Moreover, loss of heterozygosity as a result of whole Chromosome 9 loss is predicted to render the tumor deficient in TSC1 activity (Fig. 2B). There is a significant reduction in the aligned RNA-seq read coverage of TSC1 exons 9-23, downstream from the breakpoint, compared to upstream of the breakpoint, in support of the hypothesis that this tumor lacks a functional full-length copy of TSC1 (Supplemental Fig. S2).

\section{CHD7-BEND2 Rearrangement}

A second novel somatic translocation between Chromosome 8 and the $\mathrm{X}$ chromosome was detected in the genomic assembly $(t(8 ; X)(q 12.2 ; p 22.13))$ (Fig. 3A), which was also supported by the transcriptomic assembly. The rearrangement results in a novel in-frame fusion between CHD7 exons 1-2 (encoding amino acids 1-555) and BEND2 exons 5-14 (encoding amino acids 165-800) (Fig. 3B). CHD7 encodes the chromodomain helicase DNA-binding protein 7, a chromatin remodeling enzyme involved in differentiation and transcription regulation (Schnetz et al. 2010; Feng et al. 2013). Germline loss of function mutations or deletions of CHD7 are found in patients with CHARGE syndrome, a disorder characterized by dysmorphic features and congenital anomalies in multiple organs. CHD7 remodeling activity is required for neural crest cell gene expression networks, linking a lack of CHD7 activity with CHARGE syndrome features (Bajpai et al. 2010). The translocation observed in this G3 PanNET disrupts the majority of the protein-coding region of CHD7, resulting in loss of the chromodomains and helicase domain (Fig. 3B).

The CHD7 fusion partner BEND2 encodes BEN domain-containing 2, a relatively uncharacterized protein. Alignment of RNA-seq reads across the BEND2 genomic loci revealed an increase in expression of BEND2 downstream from the breakpoints, indicating the fusion of BEND2 with CHD7 enhances transcription of BEND2 (Fig. 3C). Furthermore, BEND2 was found to be an extreme high expression outlier in this tumor compared to all TCGA disease types (Fig. 3D). BEND2 protein is solely characterized by the presence of two BEN domains, which have been demonstrated to bind DNA and are typically found in proteins involved in transcription and chromatin regulation (Abhiman et al. 2008; Sathyan et al. 2011; Dai et al. 2013, 2015). Interestingly, a fusion between EWSR1 amino-terminal transactivation domain and BEND2 was identified in two patients in the ICGC study, one of which was a grade 3 PanNET (Scarpa et al. 2017). Moreover, the EWSR1-BEND2 rearrangement also similarly resulted in a fusion transcript containing exons 9-14 of BEND2, indicating a common breakpoint among these BEND2 rearrangements. In addition, a recurring fusion between MN1 and BEND2 was identified in three patients with central nervous system primitive neuroectodermal tumors (Sturm et al. 2016). This rearrangement also resulted in the juxtaposition of a transactivation domain donated by MN1 with BEND2. The cumulative evidence leads 
A

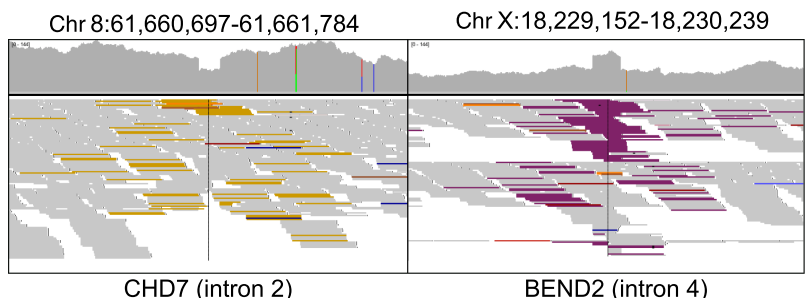

B

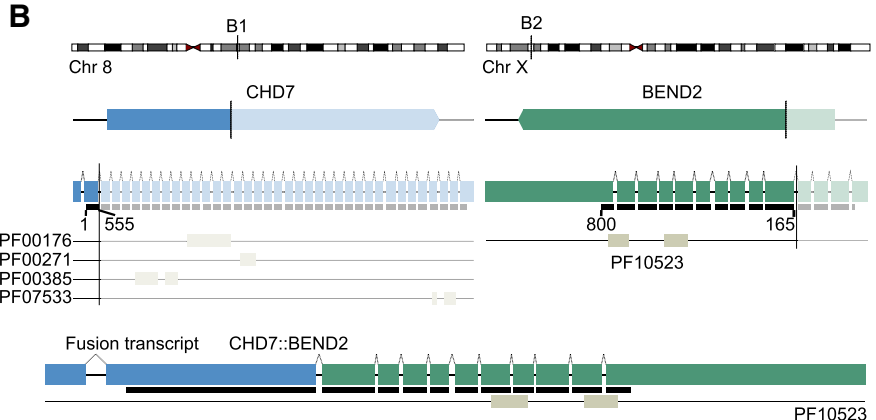

C

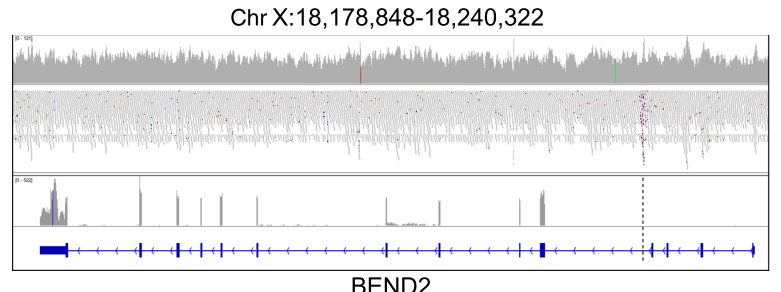

D

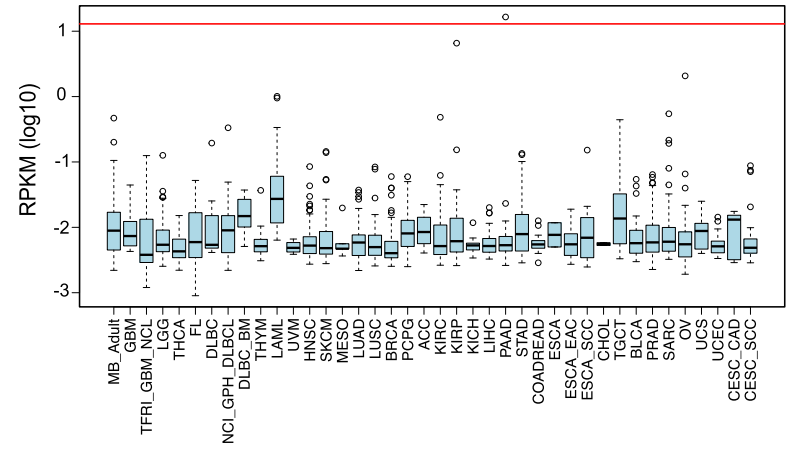

Figure 3. CHD7-BEND2 structural rearrangement and increased BEND2 expression. (A) Genomic sequencing read alignment of the genomic regions affected by the rearrangement. Gold reads that aligned to $C H D 7$ and purple reads that aligned to BEND2 indicate reads that support the rearrangement. $(B)$ Illustration of CHD7-BEND2 rearrangement. The CHD7 and BEND2 breakpoints are indicated at the chromosome, gene, and transcript level (B1 and B2, respectively, upper box). The protein-coding sequences associated with the respective transcripts are indicated by the black line, and the amino acids included in the fusion product are indicated. The predicted fusion transcript and protein-coding sequence are shown in the lower box (CHD7 exons are colored blue; BEND2 exons are colored green). Pfam protein domains are indicated by the tracks below the transcript: SNF2_N (F00176), helicase_C (PF00271), Chromo (PF00385), BRK (PF07533), BEN (PF10523). (C) Genomic sequencing reads (top) and RNA sequencing reads (bottom) aligned to the BEND2 gene. Genomic reads that are affected by the rearrangement are colored purple. The BEND2 fusion breakpoint is indicated by the dashed line. RNA-seq coverage downstream from the breakpoint (exons $5-14$ ) is increased compared to upstream of the breakpoint (exons 1-4). (D) Boxplot of BEND2 RPKM ( $\log _{10}$ transformed) across a panel of 40 TCGA data sets. BEND2 $\log _{10}(\mathrm{RPKM})$ for this patient sample is indicated by the red horizontal line.

to the hypothesis that activation of BEND2, either by fusion with a transactivation domain or potentially by increased expression, may promote oncogenic activity.

\section{Gene Expression Analysis}

RNA-seq data were compiled as gene-specific RPKM values for all seven PanNEN samples (six PanNETs and one PanNEC) sequenced as part of the POG initiative. As these samples represented several different tumor grades (two grade 1, three grade 2, and two grade 3), we evaluated the impact of tumor grade on gene expression. A mixed-effects model fit to the principal components showed that tumor grade accounted for $5 \%$ of the variability in the transcriptomic data. Based on this observation, elastic net regression was used to identify genes that could characterize tumor grade in these samples (Supplemental Fig. S3). This approach revealed genes associated with PanNEN grade. Along with the MK167, which encodes Ki-67, high expression of RIPPLY3, CIT, YBX2, and KIF19 was also associated with the G3 PanNEN signature. RIPPLY3 is expressed in islet $\beta$ cells of the pancreas and may play a role in regulating proliferation and differentiation of $\beta$ cells (Osipovich et al. 2014). Last, hierarchical clustering of the samples based on highly variable genes revealed that 
COLD SPRING HARBOR Molecular Case Studies
Genomic characterization of a G3 PanNET

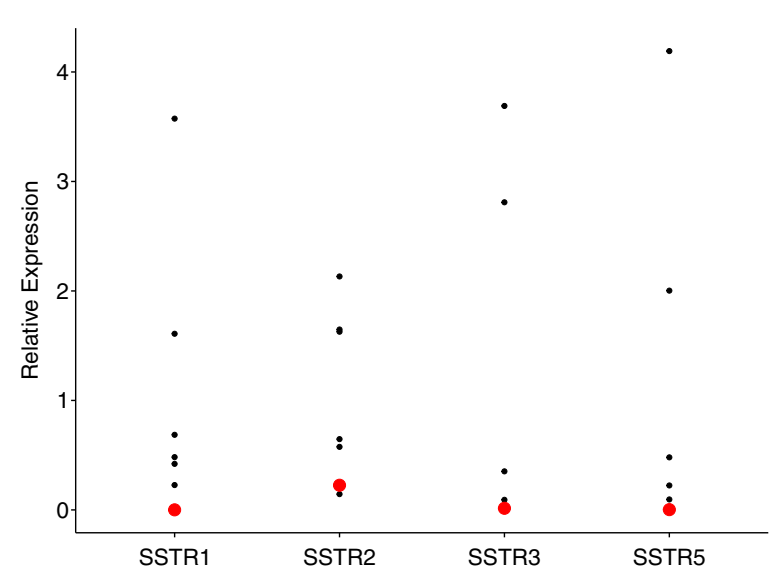

Figure 4. Low expression of SSTR genes in the G3 PanNET. SSTR1, SSTR2, SSTR3, and SSTR5 RPKM normalized to the mean of the cohort were plotted for seven metastatic PanNET samples. Relative SSTR gene expression for this patient sample is highlighted in red.

tumors with similar grade did not cluster together (Supplemental Fig. S4). These experiments suggested that tumor grade did not significantly bias global gene expression in these samples, but revealed potential gene signatures associated with tumor grade that merit further study.

Somatostatin receptors (SSTRs) are targeted by the peptide hormone somatostatin, eliciting an antiproliferative effect (Florio 2008; Rinke et al. 2009; Caplin et al. 2014). Somatostatin analogs (SSAs), such as octreotide, have played a role in the clinical setting for both tumor localization and therapeutic intervention. Given the clinical relevance of SSTRs, we investigated the expression of SSTR-encoding genes in this patient sample. There are five SSTR genes in the human genome that have been demonstrated to be heterogeneously expressed across gastro-entero-pancreatic neuroendocrine tumors (John et al. 1996; Papotti et al. 2002). To assess the relative SSTR expression in this patient sample, RNA expression of SSTR1-5 was compared among this patient sample and six additional metastatic PanNENs (Wong et al. 2018). In agreement with a previous report (Papotti et al. 2002), SSTR1 and SSTR2 were most frequently expressed across the cohort followed by SSTR3 and SSTR5 (Supplemental Fig. S5). Normalized SSTR gene expression was consistently found to be low in this patient sample compared to other PanNEN samples. This observation is in line with SSA scintillation imaging, which was negative for this patient, providing a rationale for not pursuing SSA-based therapy (Fig. 4).

\section{DISCUSSION}

\section{Single-Nucleotide and Copy-Number Variants}

Here, we describe the molecular characterization of a patient with the recently recognized distinct class of PanNEN, PanNET G3. Similar to other reports that have investigated the PanNET genomic landscape across the broad spectrum of classifications, the mutational burden of this PanNET G3 sample was relatively low compared to pancreatic ductal adenocarcinoma. Only 15 nonsynonymous SNVs and indels were identified, many of which were subclonal. Despite a high rate of occurrence in PanNETs (Jiao et al. 2011; Yachida et al. 2012; Scarpa et al. 2017), mutations or copy losses were not identified in DAXX, ATRX, or MEN1. High-grade poorly differentiated PanNECs were found to have recurrent RB1 mutations and reduced protein expression, a characteristic not frequently observed in G1 and G2 
PanNETs (Yachida et al. 2012; Bergsland et al. 2016). A single-copy loss and low expression of RB1 and homozygous loss of the upstream cell cycle-negative regulators CDKN2A and CDKN2B, which have also been observed to be mutated in a subset of PanNECs (Bergsland et al. 2016), may also contribute to the high-grade nature of this G3 PanNET.

\section{Structural Rearrangements}

Loss-of-function mutations in TSC1 and TSC2 occur in a subset of PanNETs (Jiao et al. 2011; Scarpa et al. 2017). Moreover, patients with the hereditary disorder tuberous sclerosis have an increased incidence of low-grade, well-differentiated PanNETs, further supporting a role for these tumor suppressors in PanNET oncogenesis (Larson et al. 2012). Mutations in other components of the PI3K/mTOR signaling pathway including PTEN, DEPDC5, and PIK3CA have also been observed (Jiao et al. 2011; Yachida et al. 2012; Wong et al. 2018). A novel somatic structural variant disrupting the TSC1 gene was identified in this tumor sample. The structural variant resulted in an in-frame fusion with the transmembrane protein-encoding gene, TMEM71, resulting in loss of key residues required for TSC1/2 protein complex formation. Gene fusions are more typically associated with oncogene activation as opposed to loss of function of tumor suppressors, rendering this a relatively uncommon mechanism of gene inactivation.

The mTOR inhibitor, everolimus, has been shown to significantly increase progressionfree survival in phase II and III trials and is approved for use in the PanNET setting (Yao et al. 2008, 2010, 2011, 2016). Given the incidence of mTOR pathway alterations in PanNETs, there is further rationale for treatment of patients predicted to have an aberrant mTOR pathway with mTOR inhibitors. Continued investigation will be required to determine if TSC1/TSC2-deficient PanNETs have a differential response to mTOR inhibitors compared to the other PanNETs and how this impacts response to everolimus in grade 3 well-differentiated PanNETs, which were not included in RADIANT 3 (Yao et al. 2011). The majority of reported inactivating TSC1/2 variants detected in PanNETs are SNVs or indels. The aberrant TSC1 fusion identified in this tumor highlights a potentially underappreciated mechanism of tumor-suppressor inactivation by structural rearrangement. Incorporation of structural variant detection for tumor suppressors including TSC1 and other genes associated with potentially actionable pathways may further increase the proportion of patients that could benefit from targeted therapies.

A novel rearrangement between $\mathrm{CHD7}$ and $\mathrm{BEND2}$ disrupting the majority of $\mathrm{CHD7}$ and driving expression of BEND2 was identified in this patient sample. It is unclear what the biological impact of such a fusion would have on the tumor, if any; however, recurrent fusions affecting BEND2 in this disease type indicate it may contribute to tumor biology (Scarpa et al. 2017). Although the function of the BEND2 protein has not been studied, the presence of two BEN domains suggests it may play a role in chromatin regulation and gene expression. Studies of other proteins that encode BEN domains have revealed a direct and motif-specific interaction of the BEN domain with DNA (Dai et al. 2013, 2015). Furthermore, another BEN domain-containing protein, BEND3, specifically binds heterochromatin and is proposed to contribute to transcriptional repression (Sathyan et al. 2011). Another homolog, BEND5, has been shown to similarly specifically localize to neural cortex and also stimulate transcription repression (Dai et al. 2013). Further investigation into the function of BEND2 will be required to assess the relevance of BEND2 rearrangements found in a subset of PanNETs.

\section{SSTR Expression}

SSA tumor localization was negative in this patient, indicating a lack of SSTR expression on tumor cells. In agreement with the pathology assessment, gene expression analysis of the 
five SSTR genes revealed low expression compared to a panel of previously reported metastatic PanNETs (Wong et al. 2018). Given the application of SSAs in the therapeutic setting, reduced SSTR expression as determined by lack of SSA imaging and gene expression analysis may indicate inherent resistance to SSA-based treatment.

\section{CONCLUSION}

This report represents the complete genomic and transcriptomic description of a well-differentiated grade 3 PanNET, which to date has had limited molecular characterization. Wholegenome sequencing revealed a lack of variants in the frequently mutated genes ATRX, $D A X X$, and MEN1 and identified novel structural rearrangements. Discrimination of this class of tumor in future studies will be critical for understanding the molecular mechanisms that drive their phenotypic distinction.

\section{METHODS}

\section{Sample Collection and Processing}

Following informed consent, patients underwent image-guided metastatic biopsies as part of the POG program of British Columbia (NCT02155621). Eight core biopsies using 18-22G biopsy needles were embedded in optimal cutting temperature (OCT) compound for subsequent sectioning and sequencing library preparation. Tumor sections were reviewed by a pathologist to confirm the diagnosis, evaluate tumor content and cellularity, and select areas most suitable for DNA and RNA extraction. Peripheral venous blood samples were obtained at the time of biopsy and leukocytes isolated for use as a germline reference. DNA and RNA were extracted for genomic and transcriptomic library construction, which have been previously described in detail (Jones et al. 2017).

\section{Sequencing and Bioinformatics}

Paired-end reads were generated on an Illumina HiSeq2500 sequencer and aligned to the human reference genome (GSCh37; available from http://www.bcgsc.ca/downloads/genomes/ 9606/hg19/1000genomes/bwa_ind/genome) by the BWA aligner (v0.7.6) (Li and Durbin 2010). Sequencing read lengths were $125 \mathrm{bp}$. Somatic single-nucleotide variants (SNVs) and small insertions/deletions were processed using SAMtools (Li et al. 2009), MutationSeq (v4.3.5) (Ding et al. 2012), Strelka (v1.0.6) (Saunders et al. 2012), and Trans-ABySS (v1.4.10) (Simpson et al. 2009). Regions of CNA were determined using CNAseq (v0.0.8) (Jones et al. 2010) and LOH by APOLLOH (v0.1.2) (Ha et al. 2012). Tumor content and ploidy models were estimated from sequencing data through analysis of the CNA ratios and allelic frequencies of each chromosome. This was then compared to in-house theoretical models for different ploidy at various tumor contents. Tumor content and sequencing coverage were as shown in Table 2.

Table 2. Sequencing coverage table

\begin{tabular}{lccc}
\hline Library ID & RNA/DNA & Aligned Reads & Coverage \\
\hline P02594 (Matched Normal) & DNA (PCR-free WGS) & 993322416 & $37 \times$ \\
P02600 (Tumor) & DNA (PCR-free WGS) & 2386018119 & $94 \times$ \\
P02603 (Tumor) & RNA (strand-specific) & 371042140 & \\
\hline
\end{tabular}


Structural variants were detected by de novo assembly of tumor reads using ABySS (v1.3.4) (Birol et al. 2009) and Trans-ABySS (Robertson et al. 2010), followed by variant discovery using DELLY (v0.7.3) (Rausch et al. 2012). Structural variants were visualized using MAVIS (Reisle et al. 2018). Genomic and transcriptomic sequencing read alignments were visualized using Integrative Genomics Viewer (http://software.broadinstitute.org/software/ igv/). Reads were colored by insert size and chromosome of the paired read to reveal reads that supported the structural rearrangements.

\section{Mutation Signature Analysis}

The mutation signature profile was determined by classifying genomic SNVs into 96 classes based on variant and $3^{\prime} / 5^{\prime}$ mutation context to obtain a mutation catalog vector as described by Alexandrov et al. (2013). To determine the best fit to a consensus set of 30 mutation signatures (available at http://cancer.sanger.ac.uk/cosmic/signatures), we used Markov chain Monte Carlo (MCMC) to determine the full Bayesian solution posterior of the exposure vector $\mathbf{e}$ in the multinomial mixture model $\mathbf{m}=$ Se using the sign IT package (https://github.com/ eyzhao/SignIT/) (Zhao et al. 2017). $\mathbf{S}$ is a known $96 \times 30$ matrix of consensus signatures, $\mathbf{m}$ is the 96-element mutation catalog, and the members of e denote each signature's relative contribution to the overall mutation burden. The MCMC posterior sample was examined to model the variance inherent to $\mathbf{m}$, the distributions of which are shown in Supplemental Figure S1. Where confidence intervals are reported in the text, 95\% simulated interval was reported using the 2.5 percentile and 97.5 percentile values for each signature exposure. This allows estimation of statistical confidence, with the caveat that it relies upon the underlying assumption that the 30 -signature reference set accurately reflects the space of possible signatures.

\section{Gene Expression Analysis}

RNA-seq reads were processed as previously described in Sheffield et al. (2015). Gene expression was determined as the number of reads per kilobase of transcript per million mapped reads (RPKM). For SSTR gene expression analysis, SSTR1-5 RPKM was compared across a cohort of seven PanNENs that were sequenced as part of the POG project (Wong et al. 2018). Normalized SSTR1, SSTR2, SSTR3, and SSTR5 expression was calculated by dividing RPKM by the mean RPKM for the cohort. For pan-TCGA BEND2 expression comparison, $\log _{10}$ transformed BEND2 RPKM from TCGA-sequenced tumor samples representing 40 disease and disease subtype data sets was plotted as a boxplot. BEND2 RPKM, as determined using the GAF 2.0 gene model (geneontology.org), was plotted as a red horizontal line.

The effect of tumor grade on the transcriptome was evaluated using the PVCA package in $\mathrm{R}$ (http://bioconductor.org/packages/release/bioc/html/pvca.html). Expression signatures for tumor grade were determined using elastic net regression. An elastic net model was fit to the whole transcriptome data, with the predictive outcome being tumor grade. Highly predictive features were quantified using weight analysis of the fitted model and clustered using Euclidean distance and complete hierarchical clustering.

Clustering based on gene expression (RPKM) was performed on the top 1\% variable genes selected from seven PanNENs of varying grade that were sequenced as part of the POG initiative and plotted as a heatmap. Rows (genes) and columns (samples) were clustered using the average and Manhattan methods, respectively. Tumor histology, Ki-67 staining percentage, grade, sex, and tumor content as determined by bioinformatics analysis (biofx_tc) were indicated in the horizontal tracks. 
Competing Interest Statement

The authors have declared no competing interest.

\section{Referees}

Daniel M. Halperin

Anonymous

Received November 27, 2018; accepted in revised form March 6, 2019.

\section{ADDITIONAL INFORMATION}

\section{Data Deposition and Access}

The whole-genome sequencing and RNA-seq data for this case are available as .bam files from the European Genome-phenome Archive (EGA; www.ebi.ac.uk/ega/home) as part of the study EGAS00001001159, accession ID EGAD00001004715. The TSC1-disrupting and CHD7-BEND2 gene fusions were submitted to ClinVar (https://www.ncbi.nlm .nih.gov/clinvar/) and can be found under accession numbers SCV000897763 and SCV000897764, respectively.

\section{Ethics Statement}

Patients provided written informed consent for metastatic biopsies, sequencing, and publication of results as part of the Personalized OncoGenomics Program of British Columbia (NCT 02155621, University of British Columbia Clinical Research Ethics Board approval no. H14-00681).

\section{Acknowledgments}

We gratefully acknowledge the participation of our patients and families, the POG team, and the generous support of the BC Cancer Foundation. We also acknowledge contributions toward equipment and infrastructure from Canada Foundation for Innovation and the BC Knowledge Development Fund. The results published here are in part based on data generated by the following projects and obtained from dbGaP (http://www.ncbi.nlm.nih.gov/ gap): The Cancer Genome Atlas managed by the NCl and NHGRI (http://cancergenome .nih.gov) and the Genotype-Tissue Expression (GTEx) Project supported by the Common Fund of the Office of the Director of the National Institutes of Health (https://commonfund .nih.gov/GTEx).

\section{Funding}

Funding, under the form of unrestricted philanthropic donations, was generously provided by the BC Cancer Foundation (NRF10229) and Genome British Columbia (project B20POG). We also acknowledge contributions toward equipment and infrastructure from Genome Canada and Genome British Columbia (projects 202SEQ, 212SEQ, 12002), Canada Foundation for Innovation (projects 20070, 30198, 30981, 33408), CIHR Foundation (FDN-143288) programs, and the BC Knowledge Development Fund. Additionally, M.L.T. is supported by the University of British Columbia Clinician Investigator Program.

\section{REFERENCES}

Abhiman S, lyer LM, Aravind L. 2008. BEN: a novel domain in chromatin factors and DNA viral proteins. Bioinformatics 24: 458-461. doi:10.1093/bioinformatics/btn007

Alexandrov LB, Nik-Zainal S, Wedge DC, Campbell PJ, Stratton MR. 2013. Deciphering signatures of mutational processes operative in human cancer. Cell Rep 3: 246-259. doi:10.1016/j.celrep.2012.12.008

Bajpai R, Chen DA, Rada-Iglesias A, Zhang J, Xiong Y, Helms J, Chang CP, Zhao Y, Swigut T, Wysocka J. 2010. CHD7 cooperates with PBAF to control multipotent neural crest formation. Nature 463: 958-962. doi:10 .1038/nature08733

Bergsland EK, Roy R, Stephens P, Ross JS, Bailey M, Olshen A. 2016. Genomic profiling to distinguish poorly differentiated neuroendocrine carcinomas arising in different sites. J Clin Oncol 34: 4020. doi:10.1200/ JCO.2016.34.15_suppl.4020 
Birol I, Jackman SD, Nielsen CB, Qian JQ, Varhol R, Stazyk G, Morin RD, Zhao Y, Hirst M, Schein JE, et al. 2009. De novo transcriptome assembly with ABySS. Bioinformatics 25: 2872-2877. doi:10.1093/bioinformatics/ btp367

Caplin ME, Pavel M, Ćwikla JB, Phan AT, Raderer M, Sedláčková E, Cadiot G, Wolin EM, Capdevila J, Wall L, et al. 2014. Lanreotide in metastatic enteropancreatic neuroendocrine tumors. N Engl J Med 371: 224233. doi:10.1056/NEJMoa1316158

Castro AF, Rebhun JF, Clark GJ, Quilliam LA. 2003. Rheb binds tuberous sclerosis complex 2 (TSC2) and promotes S6 kinase activation in a rapamycin- and farnesylation-dependent manner. J Biol Chem 278: 32493 32496. doi:10.1074/jbc.C300226200

Coriat R, Walter T, Terris B, Couvelard A, Ruszniewski P. 2016. Gastroenteropancreatic well-differentiated grade 3 neuroendocrine tumors: review and position statement. Oncologist 21: 1191-1199. doi:10 .1634/theoncologist.2015-0476

Dai Q, Ren A, Westholm JO, Serganov AA, Patel DJ, Lai EC. 2013. The BEN domain is a novel sequence-specific DNA-binding domain conserved in neural transcriptional repressors. Genes Dev 27: 602-614. doi:10 $.1101 / \mathrm{gad} .213314 .113$

Dai Q, Ren A, Westholm JO, Duan H, Patel DJ, Lai EC. 2015. Common and distinct DNA-binding and regulatory activities of the BEN-solo transcription factor family. Genes Dev 29: 48-62. doi:10.1101/gad.252122 114

Dasari A, Shen C, Halperin D, Zhao B, Zhou S, Xu Y, Shih T, Yao JC. 2017. Trends in the incidence, prevalence, and survival outcomes in patients with neuroendocrine tumors in the United States. JAMA Oncol 3: 13351342. doi:10.1001/jamaoncol.2017.0589

Ding J, Bashashati A, Roth A, Oloumi A, Tse K, Zeng T, Haffari G, Hirst M, Marra MA, Condon A, et al. 2012. Feature-based classifiers for somatic mutation detection in tumour-normal paired sequencing data. Bioinformatics 28: 167-175. doi:10.1093/bioinformatics/btr629

Edge S, Byrd DR, Compton CC, Fritz AG, Greene FL, Trotti A. 2010. AJCC Cancer Staging Manual. Springer, France.

Feng W, Khan MA, Bellvis P, Zhu Z, Bernhardt O, Herold-Mende C, Liu HK. 2013. The chromatin remodeler CHD7 regulates adult neurogenesis via activation of SoxC transcription factors. Cell Stem Cell 13: 6272. doi:10.1016/j.stem.2013.05.002

Florio T. 2008. Molecular mechanisms of the antiproliferative activity of somatostatin receptors (SSTRs) in neuroendocrine tumors. Front Biosci 13: 822-840. doi:10.2741/2722

Ha G, Roth A, Lai D, Bashashati A, Ding J, Goya R, Giuliany R, Rosner J, Oloumi A, Shumansky K, et al. 2012. Integrative analysis of genome-wide loss of heterozygosity and monoallelic expression at nucleotide resolution reveals disrupted pathways in triple-negative breast cancer. Genome Res 22: 1995-2007. doi:10 $.1101 /$ gr.137570.112

Huang J, Manning BD. 2008. The TSC1-TSC2 complex: a molecular switchboard controlling cell growth. Biochem J 412: 179-190. doi:10.1042/BJ20080281

Inoki K, Li Y, Xu T, Guan KL. 2003. Rheb GTPase is a direct target of TSC2 GAP activity and regulates mTOR signaling. Genes Dev 17: 1829-1834. doi:10.1101/gad.1110003

Jensen RT, Berna MJ, Bingham DB, Norton JA. 2008. Inherited pancreatic endocrine tumor syndromes: advances in molecular pathogenesis, diagnosis, management, and controversies. Cancer 113: 1807-1843. doi:10.1002/cncr.23648

Jiao Y, Shi C, Edil BH, de Wilde RF, Klimstra DS, Maitra A, Schulick RD, Tang LH, Wolfgang CL, Choti MA, et al. 2011. DAXX/ATRX, MEN1, and mTOR pathway genes are frequently altered in pancreatic neuroendocrine tumors. Science 331: 1199-1203. doi:10.1126/science.1200609

John M, Meyerhof W, Richter D, Waser B, Schaer JC, Scherübl H, Boese-Landgraf J, Neuhaus P, Ziske C, Mölling K, et al. 1996. Positive somatostatin receptor scintigraphy correlates with the presence of somatostatin receptor subtype 2. Gut 38: 33-39. doi:10.1136/gut.38.1.33

Jones SJ, Laskin J, Li YY, Griffith OL, An J, Bilenky M, Butterfield YS, Cezard T, Chuah E, Corbett R, et al. 2010. Evolution of an adenocarcinoma in response to selection by targeted kinase inhibitors. Genome Biol 11: R82. doi:10.1186/gb-2010-11-8-r82

Jones MR, Lim H, Shen Y, Pleasance E, Ch'ng C, Reisle C, Leelakumari S, Zhao C, Yip S, Ho J, et al. 2017. Successful targeting of the NRG1 pathway indicates novel treatment strategy for metastatic cancer. Ann Oncol 28: 3092-3097. doi:10.1093/annonc/mdx523

Klimstra DS, Modlin IR, Coppola D, Lloyd RV, Suster S. 2010. The pathologic classification of neuroendocrine tumors: a review of nomenclature, grading, and staging systems. Pancreas 39: 707-712. doi:10.1097/MPA $.0 \mathrm{~b} 013 \mathrm{e} 3181 \mathrm{ec} 124 \mathrm{e}$

Kulke MH, Siu LL, Tepper JE, Fisher G, Jaffe D, Haller DG, Ellis LM, Benedetti JK, Bergsland EK, Hobday TJ, et al. 2011. Future directions in the treatment of neuroendocrine tumors: consensus report of the National Cancer Institute Neuroendocrine Tumor clinical trials planning meeting. J Clin Oncol 29: 934-943. doi:10 .1200/JCO.2010.33.2056 
Larson AM, Hedgire SS, Deshpande V, Stemmer-Rachamimov AO, Harisinghani MG, Ferrone CR, Shah U, Thiele EA. 2012. Pancreatic neuroendocrine tumors in patients with tuberous sclerosis complex. Clin Genet 82: 558-563. doi:10.1111/j.1399-0004.2011.01805.x

Laskin J, Jones S, Aparicio S, Chia S, Ch'ng C, Deyell R, Eirew P, Fok A, Gelmon K, Ho C, et al. 2015. Lessons learned from the application of whole-genome analysis to the treatment of patients with advanced cancers. Cold Spring Harb Mol Case Stud 1: a000570. doi:10.1101/mcs.a000570

Li H, Durbin R. 2010. Fast and accurate long-read alignment with Burrows-Wheeler transform. Bioinformatics 26: 589-595. doi:10.1093/bioinformatics/btp698

Li H, Handsaker B, Wysoker A, Fennell T, Ruan J, Homer N, Marth G, Abecasis G, Durbin R, 1000 Genome Project Data Processing Subgroup. 2009. The Sequence Alignment/Map format and SAMtools. Bioinformatics 25: 2078-2079. doi:10.1093/bioinformatics/btp352

Lloyd RV, Osamura RY, Klöppel G, Rosai J. 2017. WHO classification of tumours of endocrine organs. IARC Press, France.

Osipovich AB, Long Q, Manduchi E, Gangula R, Hipkens SB, Schneider J, Okubo T, Stoeckert CJ, Takada S, Magnuson MA. 2014. Insm 1 promotes endocrine cell differentiation by modulating the expression of a network of genes that includes Neurog3 and Ripply3. Development 141: 2939-2949. doi:10.1242/dev .104810

Papotti M, Bongiovanni M, Volante M, Allia E, Landolfi S, Helboe L, Schindler M, Cole SL, Bussolati G. 2002. Expression of somatostatin receptor types 1-5 in 81 cases of gastrointestinal and pancreatic endocrine tumors. A correlative immunohistochemical and reverse-transcriptase polymerase chain reaction analysis. Virchows Arch 440: 461-475. doi:10.1007/s00428-002-0609-x

Rausch T, Zichner T, Schlattl A, Stutz AM, Benes V, Korbel JO. 2012. DELLY: structural variant discovery by integrated paired-end and split-read analysis. Bioinformatics 28: i333-i339. doi:10.1093/bioinformatics/ bts378

Reisle C, Mungall KL, Choo C, Paulino D, Bleile DW, Muhammadzadeh A, Mungall AJ, Moore RA, Shlafman I, Coope R, et al. 2018. MAVIS: merging, annotation, validation, and illustration of structural variants. Bioinformatics. doi:10.1093/bioinformatics/bty621

Rinke A, Müller HH, Schade-Brittinger C, Klose KJ, Barth P, Wied M, Mayer C, Aminossadati B, Pape UF, Bläker $\mathrm{M}$, et al. 2009. Placebo-controlled, double-blind, prospective, randomized study on the effect of octreotide LAR in the control of tumor growth in patients with metastatic neuroendocrine midgut tumors: a report from the PROMID Study Group. J Clin Oncol 27: 4656-4663. doi:10.1200/JCO.2009.22.8510

Robertson G, Schein J, Chiu R, Corbett R, Field M, Jackman SD, Mungall K, Lee S, Okada HM, Qian JQ, et al. 2010. De novo assembly and analysis of RNA-seq data. Nat Methods 7: 909-912.

Santiago Lima AJ, Hoogeveen-Westerveld M, Nakashima A, Maat-Kievit A, van den Ouweland A, Halley D, Kikkawa U, Nellist M. 2014. Identification of regions critical for the integrity of the TSC1-TSC2-TBC1D7 complex. PLoS One 9: e93940. doi:10.1371/journal.pone.0093940

Sathyan KM, Shen Z, Tripathi V, Prasanth KV, Prasanth SG. 2011. A BEN-domain-containing protein associates with heterochromatin and represses transcription. J Cell Sci 124: 3149-3163. doi:10.1242/jcs .086603

Saunders CT, Wong WS, Swamy S, Becq J, Murray LJ, Cheetham RK. 2012. Strelka: accurate somatic smallvariant calling from sequenced tumor-normal sample pairs. Bioinformatics 28: 1811-1817. doi:10.1093/ bioinformatics/bts271

Scarpa A, Chang DK, Nones K, Corbo V, Patch AM, Bailey P, Lawlor RT, Johns AL, Miller DK, Mafficini A, et al. 2017. Whole-genome landscape of pancreatic neuroendocrine tumours. Nature 543: 65-71. doi:10.1038/ nature21063

Schnetz MP, Handoko L, Akhtar-Zaidi B, Bartels CF, Pereira CF, Fisher AG, Adams DJ, Flicek P, Crawford GE, Laframboise T, et al. 2010. CHD7 targets active gene enhancer elements to modulate ES cell-specific gene expression. PLoS Genet 6: e1001023. doi:10.1371/journal.pgen.1001023

Sheffield BS, Tinker AV, Shen Y, Hwang H, Li-Chang HH, Pleasance E, Ch'ng C, Lum A, Lorette J, McConnell YJ, et al. 2015. Personalized oncogenomics: clinical experience with malignant peritoneal mesothelioma using whole genome sequencing. PLoS One 10: e0119689. doi:10.1371/journal.pone.0119689

Simpson JT, Wong K, Jackman SD, Schein JE, Jones SJ, Birol I. 2009. ABySS: a parallel assembler for short read sequence data. Genome Res 19: 1117-1123. doi:10.1101/gr.089532.108

Sturm D, Orr BA, Toprak UH, Hovestadt V, Jones DTW, Capper D, Sill M, Buchhalter I, Northcott PA, Leis I, et al. 2016. New brain tumor entities emerge from molecular classification of CNS-PNETs. Cell 164: 1060-1072. doi:10.1016/j.cell.2016.01.015

Tang LH, Basturk O, Sue JJ, Klimstra DS. 2016. A practical approach to the classification of WHO grade 3 (G3) well-differentiated Neuroendocrine Tumor (WD-NET) and Poorly Differentiated Neuroendocrine Carcinoma (PD-NEC) of the pancreas. Am J Surg Pathol 40: 1192-1202. doi:10.1097/PAS .0000000000000662 
Wong HL, Yang KC, Shen Y, Zhao EY, Loree JM, Kennecke HF, Kalloger SE, Karasinska JM, Lim HJ, Mungall AJ, et al. 2018. Molecular characterization of metastatic pancreatic neuroendocrine tumors (PNETs) using whole-genome and transcriptome sequencing. Cold Spring Harb Mol Case Stud 4. doi:10.1101/mcs .a002329

Yachida S, Vakiani E, White CM, Zhong Y, Saunders T, Morgan R, de Wilde RF, Maitra A, Hicks J, Demarzo AM, et al. 2012. Small cell and large cell neuroendocrine carcinomas of the pancreas are genetically similar and distinct from well-differentiated pancreatic neuroendocrine tumors. Am J Surg Pathol 36: 173-184. doi:10 .1097/PAS.0b013e3182417d36

Yao JC, Phan AT, Chang DZ, Wolff RA, Hess K, Gupta S, Jacobs C, Mares JE, Landgraf AN, Rashid A, et al. 2008. Efficacy of RAD001 (everolimus) and octreotide LAR in advanced low- to intermediate-grade neuroendocrine tumors: results of a phase II study. J Clin Oncol 26: 4311-4318. doi:10.1200/JCO.2008.16.7858

Yao JC, Lombard-Bohas C, Baudin E, Kvols LK, Rougier P, Ruszniewski P, Hoosen S, St. Peter J, Haas T, Lebwohl D, et al. 2010. Daily oral everolimus activity in patients with metastatic pancreatic neuroendocrine tumors after failure of cytotoxic chemotherapy: a phase II trial. J Clin Oncol 28: 69-76. doi:10.1200/JCO 2009.24.2669

Yao JC, Shah MH, Ito T, Bohas CL, Wolin EM, Van Cutsem E, Hobday TJ, Okusaka T, Capdevila J, de Vries EG, et al. 2011. Everolimus for advanced pancreatic neuroendocrine tumors. N Engl J Med 364: 514-523. doi:10.1056/NEJMoa1009290

Yao JC, Pavel M, Lombard-Bohas C, Van Cutsem E, Voi M, Brandt U, He W, Chen D, Capdevila J, de Vries EGE, et al. 2016. Everolimus for the treatment of advanced pancreatic neuroendocrine tumors: overall survival and circulating biomarkers from the randomized, phase III RADIANT-3 study. J Clin Oncol 34: 39063913. doi:10.1200/JCO.2016.68.0702

Zhao EY, Shen Y, Pleasance E, Kasaian K, Leelakumari S, Jones M, Bose P, Ch'ng C, Reisle C, Eirew P, et al. 2017. Homologous recombination deficiency and platinum-based therapy outcomes in advanced breast cancer. Clin Cancer Res 23: 7521-7530. doi:10.1158/1078-0432.CCR-17-1941 


\section{COLD SPRING HARBOR Molecular Case Studies}

\section{Genomic characterization of a well-differentiated grade 3 pancreatic neuroendocrine tumor}

Laura M. Williamson, Michael Steel, Jasleen K. Grewal, et al.

Cold Spring Harb Mol Case Stud 2019, 5: a003814

Access the most recent version at doi: $10.1101 /$ mcs.a003814
Supplementary http://molecularcasestudies.cshlp.org/content/suppl/2019/05/29/mcs.a003814.D Material C1

References This article cites 50 articles, 20 of which can be accessed free at: http://molecularcasestudies.cshlp.org/content/5/3/a003814.full.html\#ref-list-1

License This article is distributed under the terms of the Creative Commons Attribution-NonCommercial License, which permits reuse and redistribution, except for commercial purposes, provided that the original author and source are credited.

Email Alerting Receive free email alerts when new articles cite this article - sign up in the box at the Service top right corner of the article or click here. 